\title{
Evaluating system reliability using linear-exponential distribution function
}

\author{
Ghasem Ezzati ${ }^{1,2 *}$, Abbas Rasouli ${ }^{3}$ \\ ${ }^{1}$ Faculty of Science and Technology, Federation University, Mt Helen, Victoria, Australia \\ ${ }^{2}$ IEEE Student Member \\ ${ }^{3}$ Faculty of Science, University of Zanjan, Zanjan, Iran \\ *Corresponding author E-mail: g.ezzati@federation.edu.au
}

Copyright (C)2015 Ezzati et. al. This is an open access article distributed under the Creative Commons Attribution License, which permits unrestricted use, distribution, and reproduction in any medium, provided the original work is properly cited.

\begin{abstract}
Safety is a main criterion to design every system. Among various theories, which are applied to improve system safety, reliability theory is known as a powerful tool to reach higher safety levels in system design. In this paper, a compound of series and parallel systems is considered for reliability improvement. This system includes three items that two items are connected in parallel and their compound item is connected to the third item in series. It's assumed that the items are independent and their longevity follows linear-exponential distribution function. Reliability function of the mentioned system is formulated using linear-exponential distribution function. Then, three improvement methods will be applied to enhance system reliability. In each method, different sets of items will be considered for improvement and their reliability functions will be reformulated. A data analysis will be done in order to compare different improvement methods and a conclusion will be made based on the analyzed data.
\end{abstract}

Keywords: Improvement Methods, Linear-Exponential Distribution, Reliability Function, Series-Parallel Systems.

\section{Introduction}

Current industrial world has daily been facing with new systems requiring high levels of safety. In this regard, great attentions have been attracted by safety issues in the recent years.

It is reported that system importance has a direct relationship with system safety. Thus, there is an increasing need to work on system safety leading reliability theory [5].

Statistical and reliability analysis methods are also applied in system performance evaluation in a wide range of industries including mechanical, civil and electrical engineering $[7,8,13,18]$. For this purpose, different distribution functions, such as normal, lognormal, Weibull, exponential and linear-exponential distribution functions, have been considered in the literature to improve system reliability $[10,12,16,17]$.

Moreover, a Monte Carlo method is introduced in the literature to estimate the failure probability of a system. A multi-state criticality analysis in multi-state systems has been done to improve reliability [6]. Further, failure data has been analyzed to provide new reliability improvement methods [2].

In this paper, we apply linear-exponential distribution function in a system to investigate system reliability. The mentioned system is also considered in the literature as a radar system of an aircraft [15].

Linear-exponential distribution function is a commonly used probability distribution for modeling lifetime data and also phenomenon with linearly increasing failure rates [17]. Exponential and Rayleigh distributions are known as sub-models of linear exponential distribution function [10, 17]. 
Three improvement methods are often considered for system reliability improvement. These methods can be summarized as below $[4,15]$ :

1. Reduction Method (RM): In this method, it's considered that failure parameters of item(s) are reduced by multiplying in a factor $\rho$ where $0<\rho<1$.

2. Warm Duplication Method (WDM): A selected subset of system items will be warm duplicated in this method. In other words, item(s) selected for improvement must be added to itself (themselves) in parallel.

3. Cold Duplication Method (CDM): The only difference between this method and WDM is that a switch is used to add the spare item(s) to the system. So, the spare item will be activated when the main item is failed, while in warm duplication method new item is directly added to the main item and is active.

Organization of this paper is as follows. In the next section, reliability function (RF) will generally be explained incorporating the studied system in this paper. Then, system reliability function and its improvements will be reformulated using linear-exponential distribution. After that, a data analysis of different experiments will be done. This paper will be finished by a conclusion based on the analyzed data.

\section{Reliability function of basic complex system}

One of the most important concerns in designing systems is safety. This concern is defined using a function that is called reliability function $(\mathrm{RF})[3,5]$.

To define a reliability function of a system, $n$ independent items are often considered. If $v(t)$ was supposed as a subset of all items which have had no fault until time $t$, then $\frac{v(t)}{n}$ is a random variable showing system reliability when $n$ tends to infinity; i.e. $R(t)=\lim _{n \rightarrow \infty}\left(\frac{v(t)}{n}\right)[5]$.

In other words, reliability is a probability with which an item works over a time period $(t)$. Thus, reliability function (RF) is also supposed as below [3]:

$R(t)=P(T \geq t)$

where $T$ is a continuous random variable indicating time. Therefore, a mathematically definition of RF is as follows:

$R(t)=\int_{t}^{\infty} f(u) d u$

where $f(u)$ is probability density function (PDF).

Reliability functions are always supposed as high importance issues in designing electrical systems. These systems are basically considered as series or parallel systems. RF definition of series systems is as following [3, 5]:

$R_{S}(t)=\prod_{i=1}^{n} R_{i}(t)$

where $R_{i}(t)$ is reliability function of item $i$. A series system works when all items are working.

Also, reliability function of a parallel system is defined as below $[3,5]$ :

$R_{P}(t)=1-\prod_{i=1}^{n} Q_{i}(t)$

where $Q_{i}(t)$ is failure function of item $i$. Failure function is often supposed as a complementary function of the RF (i.e. $Q_{i}(t)=1-R_{i}(t)$ ). A parallel system works when at least one item is working.

In this paper, an electrical system is considered for reliability improvement. This system, which is supposed as a radar system in the literature [15], is shown in the Figure (1).

Reliability function of this system is formulated as below [15]:

$R(t)=\left(e^{\lambda_{2} t}+e^{\lambda_{3} t}-1\right) e^{-\Lambda t}$

where $\Lambda=\lambda_{1}+\lambda_{2}+\lambda_{3}$. This system is improved using different methods and all relevant reliability functions are formulated. 


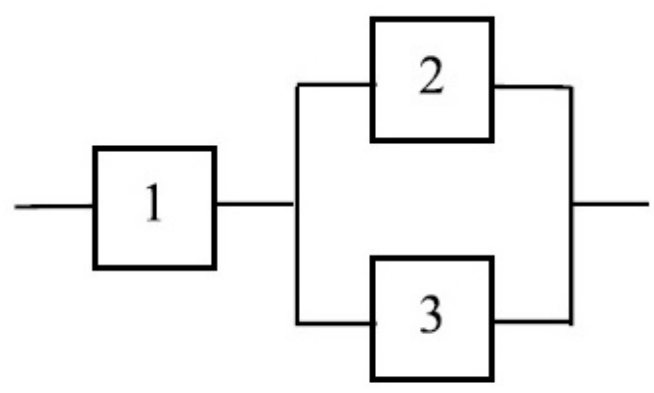

Figure 1: Basic Complex System

In this paper, we apply linear-exponential distribution function into reliability function of this system. Then, the mentioned improvement methods (reduction method, warm duplication method and cold duplication method) are considered to enhance reliability of this system.

In each improvement method, the following sets of system items are often considered for improvement [15]:

$$
\begin{aligned}
& A_{1}=\{1\} \\
& A_{2}=\{2\} \\
& A_{3}=\{1,2\} \\
& A_{4}=\{2,3\}
\end{aligned}
$$

Reliability function ( $\mathrm{RF}$ ) of this system and three improvement cases will be formulated in the next section using linear-exponential distribution function.

\section{Reliability improvements based on linear-exponential distribution}

It's supposed in this paper that random variables of the basic complex system, displayed in the Figure (1), are linear-exponentially distributed.

In general, if $t$ was a linear-exponentially distributed random variable, then its probability density function $(\mathrm{PDF})$ and reliability function $(\mathrm{RF})$ are defined, respectively, as below [10]:

$$
\begin{aligned}
& f(t)=(a+2 b t) e^{-\left(a t+b t^{2}\right)} \\
& R(t)=e^{-\left(a t+b t^{2}\right)}
\end{aligned}
$$

where $a$ and $b$ are positive constants known as failure parameters.

Furthermore, reliability function of the above mentioned system is generally written as:

$R(t)=R_{1}(t) \cdot R_{4}(t)$

where $R_{1}(t)$ is reliability function of item 1 and $R_{4}(t)$ is reliability function of the compound item (combination of items 2 and 3). Therefore, this reliability function is rewritten as below:

$R(t)=R_{1}(t) \cdot\left[R_{2}(t)+R_{3}(t)-R_{2}(t) \cdot R_{3}(t)\right]$

Thus, it can simply be shown based on the equation (7) that if random variables of the system were linearexponentially distributed, reliability function of this system is obtained as following:

$R(t)=\left[e^{a_{2} t+b_{2} t^{2}}+e^{a_{3} t+b_{3} t^{2}}-1\right] e^{-\left(\alpha t+\beta t^{2}\right)}$

where $\alpha=a_{1}+a_{2}+a_{3}$ and $\beta=b_{1}+b_{2}+b_{3}$. Also, $a_{i}$ and $b_{i}$ are failure parameters corresponding item $i$. 
Moreover, the above reliability function can be reformulated when the system is improved using either improvement method. If an item of the system was improved using the reduction method (RM), its reliability function would be as below:

$R_{i}^{\rho}(t)=e^{-\rho\left(a_{i} t+b_{i} t^{2}\right)}$

where $i$ indicates the reduced item, $a_{i}$ and $b_{i}$ are failure parameters of the selected item and $\rho$ is reduction factor which $0<\rho<1$.

Also, if $R_{i}^{W}(t)$ is the reliability function of a warm duplicated item, then its reliability function is written as following:

$R_{i}^{W}(t)=1-Q_{i}^{2}(t)=R_{i}(t)\left(2-R_{i}(t)\right)=e^{-\left(a_{i} t+b_{i} t^{2}\right)}\left(2-e^{-\left(a_{i} t+b_{i} t^{2}\right)}\right)$

Further, if an item is cold duplicated, then its reliability function would be as follows:

$R_{i}^{C}(t)=\left(1+a_{i} t+b_{i} t^{2}\right) R_{i}(t)=\left(1+a_{i} t+b_{i} t^{2}\right) e^{-\left(a_{i} t+b_{i} t^{2}\right)}$

where $R_{i}^{C}(t)$ is reliability function of the cold duplicated item.

It must be mentioned that mean time to failure (mttf) of the system and its improvements can not be derived analytically and explicitly when random variables are linear-exponentially distributed. Numerical methods should be considered to find the means [17].

The following subsections are devoted to find reliability functions of the above mentioned system when four different sets are considered for each improvement method.

\subsection{Reduction Method}

The reduction method $(\mathrm{RM})$ is applied to improve system reliability in this subsection. In this method, failure parameters of any selected subset of items must be multiplied with a factor $\rho$ that $0<\rho<1$. below:

If $A_{1}=\{1\}$ is selected for reliability improvement, then reliability function of improved system would be as

$R_{A_{1}}^{\rho}(t)=R_{1}^{\rho}(t)\left[R_{2}(t)+R_{3}(t)-R_{2}(t) \cdot R_{3}(t)\right]$

where $R_{1}^{\rho}(t)$ is reliability function of reduced component. Also, $R_{2}(t)$ and $R_{3}(t)$ are the main reliability functions of components 2 and 3 , respectively. Thus, we have:

$R_{A_{1}}^{\rho}(t)=e^{-\rho\left(a_{1} t+b_{1} t^{2}\right)}\left[e^{-\left(a_{2} t+b_{2} t^{2}\right)}+e^{-\left(a_{3} t+b_{3} t^{2}\right)}-e^{-\left(\left(a_{2}+a_{3}\right) t+\left(b_{2}+b_{3}\right) t^{2}\right)}\right]$

When the second subset $\left(A_{2}=\{2\}\right)$ is considered for improvement, RF of improved system is obtained as follows:

$R_{A_{2}}^{\rho}(t)=R_{1}(t)\left[R_{2}^{\rho}(t)+R_{3}(t)-R_{2}^{\rho}(t) \cdot R_{3}(t)\right]$

where $R_{2}^{\rho}(t)$ is reliability function of second component. Hence, it can be concluded that:

$R_{A_{2}}^{\rho}(t)=e^{-\left(a_{1} t+b_{1} t^{2}\right)}\left[e^{-\rho\left(a_{2} t+b_{2} t^{2}\right)}+e^{-\left(a_{3} t+b_{3} t^{2}\right)}-e^{-\left(\rho\left(a_{2} t+b_{2} t^{2}\right)+\left(a_{3} t+b_{3} t^{2}\right)\right)}\right]$

Also, if $A_{3}=\{1,2\}$ is supposed for reliability improvement, then the improved system will have a reliability function as:

$R_{A_{3}}^{\rho}(t)=R_{1}^{\rho}(t)\left[R_{2}^{\rho}(t)+R_{3}(t)-R_{2}^{\rho}(t) \cdot R_{3}(t)\right]$

and then we'll have:

$R_{A_{3}}^{\rho}(t)=e^{-\rho\left(a_{1} t+b_{1} t^{2}\right)}\left[e^{-\rho\left(a_{2} t+b_{2} t^{2}\right)}+e^{-\left(a_{3} t+b_{3} t^{2}\right)}-e^{-\left(\rho\left(a_{2} t+b_{2} t^{2}\right)+\left(a_{3} t+b_{3} t^{2}\right)\right)}\right]$

Further, assuming $A_{4}=\{2,3\}$ as the selected items for improvement results the following reliability function of the improved system:

$R_{A_{4}}^{\rho}(t)=R_{1}(t)\left[R_{2}^{\rho}(t)+R_{3}^{\rho}(t)-R_{2}^{\rho}(t) \cdot R_{3}^{\rho}(t)\right]$

and it can be concluded that:

$R_{A_{4}}^{\rho}(t)=e^{-\left(a_{1} t+b_{1} t^{2}\right)}\left[e^{-\rho\left(a_{2} t+b_{2} t^{2}\right)}+e^{-\rho\left(a_{3} t+b_{3} t^{2}\right)}-e^{-\rho\left(\left(a_{2}+a_{3}\right) t+\left(b_{2}+b_{3}\right) t^{2}\right)}\right]$ 


\subsection{Warm Duplication Method}

In this subsection, the warm duplication method (WDM) is applied to improve system reliability. When an item is warm duplicated, an identical item is added to the first item in parallel. Thus, if the sets $A_{1}=\{1\}, A_{2}=\{2\}$, $A_{3}=\{1,2\}$ and $A_{4}=\{2,3\}$ are considered for reliability improvement, then the following reliability functions will be obtained, respectively:

$$
\begin{aligned}
& R_{A_{1}}^{W}(t)=R_{1}(t)\left(2-R_{1}(t)\right)\left[R_{2}(t)+R_{3}(t)-R_{2}(t) \cdot R_{3}(t)\right] \\
& R_{A_{2}}^{W}(t)=R_{1}(t)\left[R_{3}(t)+R_{2}(t)\left(2-R_{2}(t)\right)\left(1-R_{3}(t)\right)\right] \\
& R_{A_{3}}^{W}(t)=R_{1}(t)\left(2-R_{1}(t)\right)\left[R_{3}(t)+R_{2}(t)\left(2-R_{2}(t)\right)\left(1-R_{3}(t)\right)\right] \\
& R_{A_{4}}^{W}(t)=R_{1}(t)\left[R_{3}(t)\left(2-R_{3}(t)\right)+R_{2}(t)\left(2-R_{2}(t)\right)\left(1-R_{3}(t)\left(2-R_{3}(t)\right)\right)\right]
\end{aligned}
$$

where $R_{1}(t), R_{2}(t)$ and $R_{3}(t)$ are reliability functions of the items 1,2 and 3 , respectively.

Therefore, reliability functions of various improved systems with different sets of items will be obtained as below:

$$
\begin{aligned}
& R_{A_{1}}^{W}(t)=\left(2-e^{-\left(a_{1} t+b_{1} t^{2}\right)}\right)\left[e^{a_{2} t+b_{2} t^{2}}+e^{a_{3} t+b_{3} t^{2}}-1\right] e^{-\left(\alpha t+\beta t^{2}\right)} \\
& R_{A_{2}}^{W}(t)=e^{-\left(a_{1} t+b_{1} t^{2}\right)}\left[e^{-\left(a_{2} t+b_{2} t^{2}\right)}\left(1-e^{-\left(a_{3} t+b_{3} t^{2}\right)}\right)\left(2-e^{-\left(a_{2} t+b_{2} t^{2}\right)}\right)+e^{-\left(a_{3} t+b_{3} t^{2}\right)}\right] \\
& R_{A_{3}}^{W}(t)=e^{-\left(a_{1} t+b_{1} t^{2}\right)}\left(2-e^{-\left(a_{1} t+b_{1} t^{2}\right)}\right)\left[e^{-\left(a_{2} t+b_{2} t^{2}\right)}\left(1-e^{-\left(a_{3} t+b_{3} t^{2}\right)}\right)\left(2-e^{-\left(a_{2} t+b_{2} t^{2}\right)}\right)+e^{-\left(a_{3} t+b_{3} t^{2}\right)}\right] \\
& R_{A_{4}}^{W}(t)=e^{-\left(a_{1} t+b_{1} t^{2}\right)}\left[e^{-\left(a_{3} t+b_{3} t^{2}\right)}\left(2-e^{-\left(a_{3} t+b_{3} t^{2}\right)}\right)+e^{-\left(a_{2} t+b_{2} t^{2}\right)}\left(2-e^{-\left(a_{2} t+b_{2} t^{2}\right)}\right)\left(1-e^{-\left(a_{3} t+b_{3} t^{2}\right)}\left(2-e^{-\left(a_{3} t+b_{3} t^{2}\right)}\right)\right)\right]
\end{aligned}
$$

\subsection{Cold Duplication Method}

This subsection provides reliability functions of system improved by the cold duplication method (CDM). When an item is cold duplicated, this item will be duplicated by adding an identical item in parallel using a switch. In this case, the spare item is activated after failure of the first item.

Therefore, when four different sets of items are selected for improvement, their reliability functions are obtained as follows, respectively:

$$
\begin{aligned}
& R_{A_{1}}^{C}(t)=A * R_{1}(t)\left[R_{2}(t)+R_{3}(t)-R_{2}(t) \cdot R_{3}(t)\right] \\
& R_{A_{2}}^{C}(t)=R_{1}(t)\left[B * R_{2}(t)\left(1-R_{3}(t)\right)+R_{3}(t)\right] \\
& R_{A_{3}}^{C}(t)=A * R_{1}(t)\left[B * R_{2}(t)\left(1-R_{3}(t)\right)+R_{3}(t)\right] \\
& R_{A_{4}}^{C}(t)=R_{1}(t)\left[A * R_{2}(t)+C * R_{3}(t)-B * C * R_{2}(t) * R_{3}(t)\right]
\end{aligned}
$$


where $A=\left(1+a_{1} t+b_{1} t^{2}\right), B=\left(1+a_{2} t+b_{2} t^{2}\right)$ and $C=\left(1+a_{3} t+b_{3} t^{2}\right)$.

The difference between this method and the warm duplication method is to use a switch. This difference results less failure probability of a cold duplicated item than probability of failure of an improved item by the warm duplication method. Therefore, the reliability of the system will be increased.

On the other hand, the added switch can affect reliability of the whole system and decrease reliability level. So, the warm and cold duplication methods are not comparable generally.

However, reliability functions of different cold duplicated systems are shown here. These functions are obtained based on different selected sets.

$R_{A_{1}}^{C}(t)=A\left(e^{a_{2} t+b_{2} t^{2}}+e^{a_{3} t+b_{3} t^{2}}-1\right) e^{-\left(\alpha t+\beta t^{2}\right)}$

$R_{A_{2}}^{C}(t)=e^{-\left(a_{1} t+b_{1} t^{2}\right)}\left[B * e^{-\left(a_{2} t+b_{2} t^{2}\right)}\left(1-e^{-\left(a_{3} t+b_{3} t^{2}\right)}\right)+e^{-\left(a_{3} t+b_{3} t^{2}\right)}\right]$

$R_{A_{3}}^{C}(t)=A * e^{-\left(a_{1} t+b_{1} t^{2}\right)}\left[B * e^{-\left(a_{2} t+b_{2} t^{2}\right)}\left(1-e^{-\left(a_{3} t+b_{3} t^{2}\right)}\right)+e^{-\left(a_{3} t+b_{3} t^{2}\right)}\right]$

$R_{A_{4}}^{C}(t)=B * e^{-\left(\left(a_{1}+a_{2}\right) t+\left(b_{1}+b_{2}\right) t^{2}\right)}+C * e^{-\left(\left(a_{1}+a_{3}\right) t+\left(b_{1}+b_{3}\right) t^{2}\right)}-B * C * e^{-\left(\alpha t+\beta t^{2}\right)}$

where $A=\left(1+a_{1} t+b_{1} t^{2}\right), B=\left(1+a_{2} t+b_{2} t^{2}\right)$ and $C=\left(1+a_{3} t+b_{3} t^{2}\right)$.

Several numerical experiments will be solved in the next section in order to make a better sense of reliability of a system and its improvements when random variables are linear-exponentially distributed.

\section{Data analysis}

In this section, reliability function of the mentioned complex system will be formulated for special cases, before improvement and after that.

At first, reliability function of the original system will be formulated when random variables are linear-exponentially distributed.

Then, the improvement methods will be applied to enhance the system reliability and hence reliability functions will be reformulated based on different improvement methods.

Number of experiments are provided in the following subsection in order to discuss about different improvement methods. A conclusion based on the obtained numerical results will be provided in the next section.

\subsection{Reduction Method for Different Items}

The basic complex system is improved in this subsection using reduction method (RM). Four different sets of items are selected for improvement.

Suppose that the following failure parameter are given:

$a_{1}=0.8, b_{1}=1.1, a_{2}=0.65, b_{2}=0.9, a_{3}=0.85, b_{3}=1.25$

Also, it's supposed that $\rho=0.6$. Thus, based on the equation (8), original reliability function of the complex system would be as below:

$R(t)=e^{-\left(1.45 t+2 t^{2}\right)}+e^{-\left(1.65 t+2.35 t^{2}\right)}-e^{-\left(2.3 t+3.25 t^{2}\right)}$

Therefore, if different sets are selected for improvement, the following reliability functions are obtained for the improved systems, respectively:

$$
\begin{aligned}
& R_{A_{1}}^{\rho}(t)=e^{-\left(1.13 t+1.56 t^{2}\right)}+e^{-\left(1.33 t+1.91 t^{2}\right)}-e^{-\left(1.98 t+2.71 t^{2}\right)} \\
& R_{A_{2}}^{\rho}(t)=e^{-\left(1.19 t+1.64 t^{2}\right)}+e^{-\left(1.65 t+2.35 t^{2}\right)}-e^{-\left(2.04 t+2.89 t^{2}\right)} \\
& R_{A_{3}}^{\rho}(t)=e^{-\left(0.87 t+1.2 t^{2}\right)}+e^{-\left(1.33 t+1.91 t^{2}\right)}-e^{-\left(1.72 t+2.45 t^{2}\right)}
\end{aligned}
$$


$R_{A_{4}}^{\rho}(t)=e^{-\left(1.19 t+1.64 t^{2}\right)}+e^{-\left(1.31 t+1.85 t^{2}\right)}-e^{-\left(1.7 t+2.39 t^{2}\right)}$

Graphs of reliability functions of the original and improved systems are shown in the Figure (2).

It can clearly be seen in the figure that the reduction method has a consistent trend to improve system reliability in this problem. Also, all improved systems have higher reliability than the original system that is acceptable. In this case, $A_{3}=\{1,2\}$ reaches the best reliability level and then $A_{1}, A_{4}$ and $A_{2}$ are in the next positions, respectively.

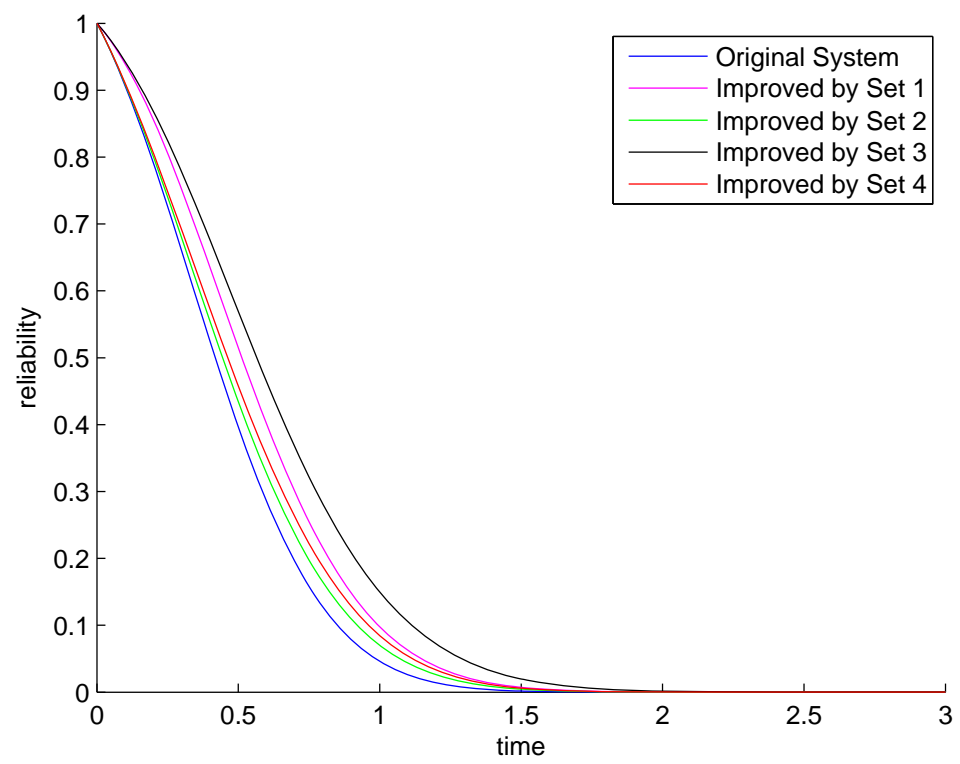

Figure 2: Reduction Method

Further, although all reliability levels finally tend to zero, the main RF tends to zero at $t=2.2215$ while improved RF by the third set $\left(A_{3}\right)$ tends to zero at $t=2.9435$. It can be concluded that there is a big enhancement in reliability when system is improved by the reduction method.

\subsection{Reduction Method with Different Reduction Factors}

Various reduction factors are applied in this subsection for reliability improvement. The following failure parameters are considered:

$a_{1}=0.2, b_{1}=0.3, a_{2}=0.15, b_{2}=0.1, a_{3}=0.35, b_{3}=0.5$

Thus, reliability function of the complex electrical system including three independent and identical components is as follows:

$R(t)=e^{-\left(0.55 t+0.8 t^{2}\right)}+e^{-\left(0.35 t+0.4 t^{2}\right)}-e^{-\left(0.7 t+0.9 t^{2}\right)}$

Then, a system using $A_{3}=\{1,2\}$ (as a selected set for improvement) is considered for improving using reduction method. Various reduction factors are applied to compare effects of various $\rho$ s on the system.

Three different amounts are supposed as reduction factor as below:

$\rho_{1}=0.2, \rho_{2}=0.5, \rho_{3}=0.8$

Therefore, reliability functions of improved systems using these reduction factors will respectively be as follows:

$R_{A_{3}}^{\rho_{1}}(t)=e^{-\left(0.07 t+0.08 t^{2}\right)}+e^{-\left(0.39 t+0.56 t^{2}\right)}-e^{-\left(0.42 t+0.58 t^{2}\right)}$ 
$R_{A_{3}}^{\rho_{2}}(t)=e^{-\left(0.175 t+0.2 t^{2}\right)}+e^{-\left(0.45 t+0.65 t^{2}\right)}-e^{-\left(0.525 t+0.7 t^{2}\right)}$

$R_{A_{3}}^{\rho_{3}}(t)=e^{-\left(0.28 t+0.32 t^{2}\right)}+e^{-\left(0.51 t+0.74 t^{2}\right)}-e^{-\left(0.63 t+0.82 t^{2}\right)}$

Figure (3) shows four different graphs for main system and also the improve systems by different reduction factors.

When reduction factor $\rho$ tends to zero, reliability improvement is increased. It's displayed in the Figure (3) that the best reliability is obtained when $\rho=0.2$. Second and third reliability factors $(0.5$ and 0.8$)$ reach second and third reliability levels, respectively.

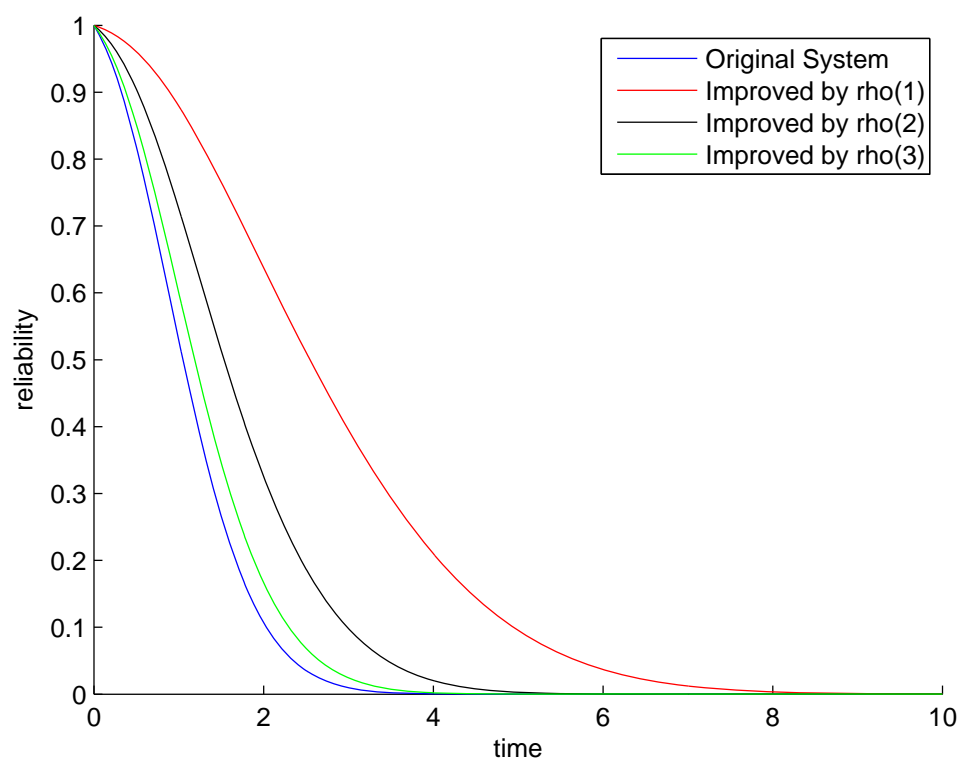

Figure 3: Different Reduction Factors

As the previous experiment, trends of reliability functions are consistent. Also, it can be seen that there are big differences between reliability functions because of bid differences between the given reduction factors. It can be concluded from the Figure (3) that system remains safe in a longer time when the reduction factor is smaller.

\subsection{Comparing Different Improvement Methods}

In this subsection, different improvement methods are applied to improve reliability of the mentioned system using just the first component. The following data are supposed in this experiment:

$a_{1}=0.45, b_{1}=0.25, a_{2}=0.5, b_{2}=0.7, a_{3}=0.65, b_{3}=0.15$

So, reliability function of the complex system is as below:

$R(t)=e^{-\left(0.95 t+0.95 t^{2}\right)}+e^{-\left(1.1 t+0.4 t^{2}\right)}-e^{-\left(1.6 t+1.1 t^{2}\right)}$

If the system is improved using the reduction method where $\rho=0.5$, then its reliability function is obtained as follows:

$R_{A_{1}}^{\rho}(t)=e^{-\left(0.725 t+0.825 t^{2}\right)}+e^{-\left(0.875 t+0.275 t^{2}\right)}-e^{-\left(1.375 t+0.975 t^{2}\right)}$

Moreover, when the first item of system is duplicated using ward and cold duplication methods, the following reliability functions are obtained, respectively:

$R_{A_{1}}^{W}(t)=\left(2-e^{-\left(0.45 t+0.25 t^{2}\right)}\right)\left[e^{-\left(0.95 t+0.95 t^{2}\right)}+e^{-\left(1.1 t+0.4 t^{2}\right)}-e^{-\left(1.6 t+1.1 t^{2}\right)}\right]$ 
$R_{A_{1}}^{C}(t)=\left(1+0.45 t+0.25 t^{2}\right)\left[e^{-\left(0.95 t+0.95 t^{2}\right)}+e^{-\left(1.1 t+0.4 t^{2}\right)}-e^{-\left(1.6 t+1.1 t^{2}\right)}\right]$

Reliability function of the main system and also improved system using different methods are displayed in the Figure (4).

As can be seen in the Figure (4), trends of reliability functions are not consistent in this experiment and they intercept each other. At the beginning of the experiment, the best reliability belongs to the cold duplication method. In this time period (up to $t=1.2586$ ), the warm duplication method and the reduction method reach the second and third reliability levels, respectively.

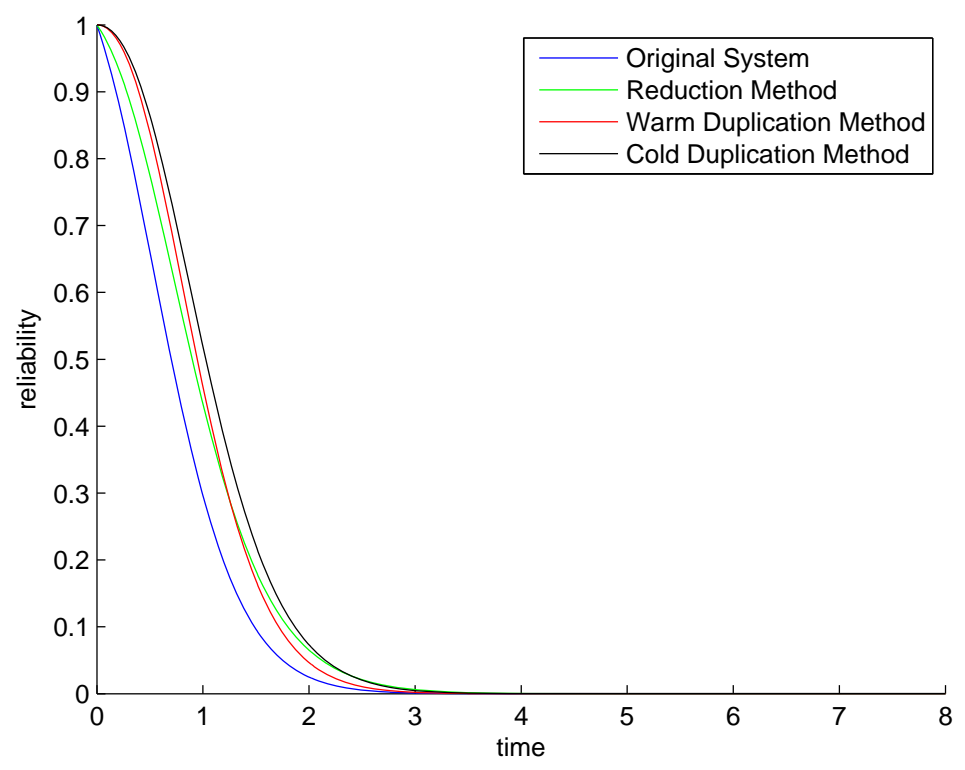

Figure 4: Different Improvement Methods

After that, the reduction method reaches a reliability higher than the warm duplication method, while the cold duplication method is still the best. This trend changes at $t=2.3957$ when the highest reliability comes from the reduction method.

It must be mentioned that at all times, the cold duplication method works better than the warm duplication method. Further, the main system (before improvement) has always the minimum reliability level. Thus, it can be resulted that different improvement methods provide various levels of reliability and also order of reliability levels is sometimes changed. One would expect is to check reliability improvements in order to select the best method providing highest reliability.

\section{Conclusion}

Reliability function (RF) of a basic complex system is studied in this paper. We have reformulated this RF considering linear-exponential distribution function.

Three improvement methods, including reduction method (RM), warm duplication method (WDM) and cold duplication method (CDM), are also applied to improve reliability of the mentioned system. It is shown that reliability of an improved system is higher than the original system reliability in all cases.

It's found that if reduction method is applied to improve system reliability using different sets of items, a combination of items 1 and 2 is the best option for reliability improvement. Also, it's concluded that when a single set of items is improved using the reduction method, the smaller reduction factor applied for reliability improvement, the higher reliability for the system. 
Further, it's shown in this paper that the cold duplication method improves system reliability much better than the warm duplication method, but it's not possible to make a general statement for a comparison between reduction method and duplication methods.

\section{Acknowledgements}

This research is partially supported by the National ICT Australia (NICTA).

\section{References}

[1] Ahi, P. and Jenab, K. and Ghasempoor, A. and Rajai, M., "A Survivability Model for Ejection of Green Compacts in Powder Metallurgy Technology", International Journal of Industrial Engineering Computations, Vol. 3 (2012), pp. $15-24$.

[2] Ascher, H., "Different Insights for Part and System Reliability Obtained from Exactly Same DFOM Failure Numbers", Reliability Engineering and System Safety, Vol. 92 (2007), pp. 552-559.

[3] Ayyub, B. M. and McCuen, R. H., Probability, Statistics and Reliability for Engineers and Scientists, Boca Raton, CRC Press, (2011).

[4] Billinton, R. and Allan, R. N., Reliability Evaluation of Engineering Systems; Concepts and Techniques Plenum Press, (1992).

[5] Birolini, A., Reliability Engineering, Theory and Pracitce, Fifth Edition Springer, Berlin, (2007).

[6] Csenki, A., "On Continuous Lifetime Distributions with Polynomial Failure Rate with an Application in Reliability", Reliability Engineering and System Safety, Vol. 96 (2011), pp. 1587-1590.

[7] Ezzati, G. and Mammadov, M. and Kulkarni, S., "A New Reliability Analysis Method based on the Conjugate Gradient Direction", Structural and Multidisciplinary Optimization, DOI: 10.1007/s00158-014-1113-z, (2014).

[8] Ezzati, G. and Mammadov, M. and Kulkarni, S., "Solving Reliability Analysis Problems in the Polar Space", International Journal of Applied Mathematical Research, Vol. 3, No. 4 (2014), pp. 353-365.

[9] Levitin, G. and Xing, L. and Ben-Haim, H. and Dai, Y., "Reliability of Series-Parallel Systems With Random Failure Propagation Time", IEEE Transactions on Reliability, Vol. 62, No. 3 (2013), pp. 637-647.

[10] Mahmoud, M. A. W. and Alam, F. M. A., "The Generalized Linear Exponential Distribution", Statistics and Probability Letters, Vol. 80 (2010), pp. 1005-1014.

[11] Mustafa, A. and El-Faheem, A. A., "Reliability Equivalence Factors of a System with Mixture of n Independent and Non-identical Lifetimes with Delay Time", Journal of Egyptian Mathematical Society, Vol. 22 (2014), pp. 96-101.

[12] Mustafa, A., "Reliability Equivalence Factors for Some Systems with Mixture Weibull Failure Rates", American Journal of Mathematics and Statistics, Vol. 2, No. 1 (2012), pp. 6-12.

[13] Pyttel, B. and Grawenhof, P. and Berger, C., "Application of Different Concepts for Fatigue Design of Welded Joints in Rotating Components in Mechanical Engineering", International Journal of Fatigue, Vol. 34 (2012), pp. 35-46.

[14] Rao, B. S. and Kanta, R. R. L. and Rosaiah, K. and Babu, M. S., "Exponential-Gamma Additive Failure Rate Model", Journal of Safety Engineering, Vol. 2, No. 2A (2013), pp. 1-6.

[15] Sarhan, A. M., "Reliability Equivalence with a Basic Series/Parallel System", Applied Mathematics and Computation, Vol. 132 (2002), pp. 115-133.

[16] Sarhan, A. M. and Apaloo, J., "Exponentiated Modified Weibull Extension Distribution", Reliability Engineering and System Safety, Vol. 112 (2013), pp. 137-144.

[17] Sarhan, A. M. and Ahmad, A. E. A. and Alasbahi, I. A., "Exponentiated Generalized Linear Exponential Distribution", Applied Mathematical Modelling, Vol. 37 (2013), pp. 2838-2849.

[18] Stapelberg, R. F., Handbook of Reliability, Availability, Maintainability and Safety in Engineering Design, Springer, (2009).

[19] Yousry, H. A. and Shawky, A. I. and Al-Ohally, M. I., "Reliability Equivalence of Independent Non-identical Parallel and Series Systems", Life Science Journal, Vol. 9, No. 3 (2012), pp. 577-583. 